\title{
Artículo
}

\section{Incidencia de las metas académicas del alumnado de secundaria en su gestión motivacional}

\author{
Iris Estevez*, Susana Rodríguez, Antonio Valle, Bibiana Regueiro e Isabel Piñeiro \\ Departamento de Psicología Evolutiva y de la Educación, Universidad de A Coruña, La Coruña, España
}

\section{INFORMACIÓN DEL ARTÍ́CULO}

\section{Historia del artículo:}

Recibido el 26 de diciembre de 2015

Aceptado el 3 de marzo de 2016

On-line el 30 de abril de 2016

\section{Palabras clave:}

Orientación a metas

Demora de la gratificación

Activación de creencias de autoeficacia

Estrategias volitivas

\begin{abstract}
R E S U M E N
Desde diversas posiciones teóricas e investigaciones recientes se enfatiza la importancia de atender a los componentes motivacionales implicados en el aprendizaje. El objetivo del presente estudio es averiguar qué orientación a metas es más característica de los estudiantes que utilizan estrategias volitivas como la demora de la gratificación o la activación de creencias de autoeficacia para el sostenimiento del compromiso con el estudio. Con una muestra de 152 estudiantes de 2 . $^{\circ}$ curso de educación secundaria obligatoria con edades comprendidas entre los 13 y los 16 años $(M=13,82)$ se empleó el análisis de regresión a fin de determinar el grado en que la estructura de metas predice el uso de estas estrategias volitivas. Los resultados muestran que trabajar por interés en la materia, para la adquisición de un trabajo futuro digno o para ser más competente podrían predecir positivamente la activación de creencias de autoeficacia. Por otro lado, y a diferencia de lo que se había hipotetizado, la demora de la gratificación se ha asociado en mayor medida a las metas de logro o recompensa. En la explicación de esta diferencial vinculación se alude al carácter más o menos autotélico que caracteriza las metas académicas.

(C) 2016 Instituto de Ciencias de la Educación de la Universidad de Oviedo. Publicado por Elsevier

España, S.L.U. Este es un artículo Open Access bajo la CC BY-NC-ND licencia (http://creativecommons. org/licencias/by-nc-nd/4.0/).

\section{Incidence of academic targets of secondary school pupils in their motivational management}

\section{A B S T R A C T}

From diverse theoretical positions and recent researches, the importance of taking care of the motivation components involved in the learning process is emphasizing. The aim of this review is to find the most characteristic goal orientation of the individuals who use the volitive strategies like the delay of gratification or the activation of the self-efficacy beliefs for the maintenance of the commitment to the study. Based on a sampling of 152 students of 2 nd year of mandatory secondary education ranged from 13 to 16 with a mean age of 13.82 , and starting with a correlation analysis, we have employed a regression analysis in order to decide the degree to which the goal structure predicts the use of these volitive strategies. The results show us that working interested in the subject, for the acquisition of a decent future job or to be more competent, could influence positively in the activation of self-efficacy beliefs. On the other hand, unlike what we had initially hypothesized, the strategy delay of gratification is more associated with goals based on achievements or rewards. Finally, we have indicated the external and internal nature of the academic goals to explain these different links.

(c) 2016 Instituto de Ciencias de la Educación de la Universidad de Oviedo. Published by Elsevier España, S.L.U. This is an open access article under the CC BY-NC-ND license (http://creativecommons. org/licenses/by-nc-nd/4.0/).

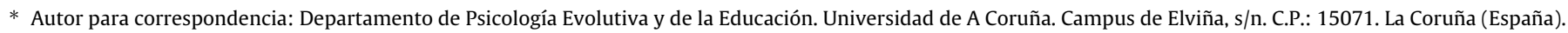
Correos electrónicos: iris.estevezb@udc.es, irisblanco14@hotmail.com (I. Estevez).
} 


\section{Introducción}

Cuando un estudiante empieza a hacer los deberes para el día siguiente, unos amigos le proponen ir al cine; su intención inicial seguramente se resiente y el estudiante tiene ahora que gestionar su motivación para hacer esos deberes. Este tipo de «trabajo cognitivo" que constantemente abordan los estudiantes es lo que se estudia bajo el tópico de gestión o regulación de la voluntad (Corno, 1993; Wolters, 2003). Si la motivación es un conjunto de procesos que ha llevado a este estudiante a identificar y seleccionar el propósito -hacer los deberes para el día siguiente-, la volición se refiere al manejo de la propia motivación con objeto de no perder de vista la meta inicial -por ejemplo, no ir al cine para poder terminar los deberes-. Cabe esperar que, dados los múltiples distractores con los que conviven los estudiantes, la habilidad para regular la propia motivación y sostener su voluntad esté fuertemente vinculada con el éxito académico (Corno, 2001; Bembenutty y Karabenick, 2004). A pesar de ello, no se sabe mucho de los factores que influyen en el uso de estrategias para sostener la voluntad y es por ello por lo que este trabajo estudia la orientación a metas como posible antecedente del empleo de diferentes estrategias para gestión de la motivación.

\section{Autorregulación motivacional del aprendizaje}

Este trabajo se fundamenta en el constructo de autorregulación inicialmente propuesto por Bandura (1991) que desde la perspectiva sociocognitiva permite diferenciar la regulación comportamental y emocional. Tomando en consideración los trabajos clásicos de Zimmerman y Schunk (1998) o Graham y Harris (1997), partimos de la diferenciación de estrategias de autorregulación en el ámbito cognitivo, motivacional y contextual (Kaplan, 2008). Concretamente, la autorregulación de la motivación implicaría el ajuste y la gestión de, al menos, los siguientes aspectos: 1) las razones para implicarse y desarrollar la actividad; 2 ) las creencias sobre su importancia, utilidad o relevancia; 3 ) los niveles de interés personal por el ámbito al que pertenece la tarea; 4) los juicios acerca de la autoeficacia para desarrollarla adecuadamente; 5) la valía personal y 6) la ansiedad y las emociones negativas asociadas a la tarea. Estos aspectos pueden observarse, tal y como se plantea en este trabajo, bajo el marco teórico de las 3 dimensiones de la motivación académica propuestas por Pintrich y de Groot (1990).

Es en esta área de la autorregulación donde adquieren toda su relevancia las estrategias para la gestión de la motivación definidas genéricamente como mecanismos y procedimientos empleados para promover estados emocionalmente adaptativos o para gestionar aquellas situaciones de riesgo que afectan al bienestar personal (García, 1995). Tradicionalmente se ha diferenciado entre estrategias volitivas, orientadas a mantener el compromiso con las intenciones de aprendizaje, y estrategias defensivas o de autoprotección (Rodríguez, González, Valle, Núñez y González-Pienda, 2004; Valle, González, Rodríguez, Núñez y González-Pienda, 2006).

\section{Estrategias volitivas}

Además de atender a los aspectos más tradicionalmente cognitivos, los estudiantes necesitan planificar e implementar estrategias que les ayuden a persistir y mantener su esfuerzo en la consecución de las metas propuestas; estrategias que se denominan volitivas, en la medida en que contribuyen a sostener la voluntad en este camino. La habilidad de una persona para mantener su intención hacia la meta -su nivel de volición- podría estar determinado por el uso de diferentes estrategias (Wolters, 1999), en la medida en que estas contribuyen a limitar el impacto de distracciones internas o externas (Pintrich y Schunk, 2006) y podrían repercutir positivamente en los niveles de persistencia y sobre el desempeño académico en secundaria (López, 2000).

Pintrich (2000) elabora una taxonomía de estrategias volitivas entre las que se ubican las 2 estrategias estudiadas en este trabajo: la activación de creencias de autoeficacia y la provisión de recompensas -demora de la gratificación-. La activación de creencias de autoeficacia, referida a procedimientos dirigidos a fortalecer la autoeficacia o la confianza en la realización de la tarea, implicaría, por ejemplo, rememorar éxitos pasados o dirigirse a uno mismo con el lema "puedo hacerlo/puedo lograrlo». La provisión de recompensas implica que el estudiante se proponga a sí mismo algún tipo de recompensa externa o estímulo extrínseco para sostener o incrementar el esfuerzo dedicado a la tarea. Esta estrategia volitiva se conoce usualmente como demora de la gratificación.

\section{Orientación a metas}

Atendiendo al objeto de esta investigación, se entiende que las metas personales constituyen un marco de referencia de gran importancia para explicar, en general, los patrones de comportamiento en el ámbito escolar y, específicamente, la gestión de la propia motivación y voluntad (Valle, Núñez, Gabanach, Rodríguez, González-Pienda y Rosario, 2009). El estudio de las metas académicas que persigue el estudiante ha cobrado especial interés en la literatura motivacional en la medida en que organizan y regulan el comportamiento de cara a la consecución de un determinado logro y reflejan los criterios mediante los cuales las personas juzgan su ejecución en la tarea y su éxito o fracaso respecto a las consecución de esas metas (Urdan, 2004).

En secundaria, trabajos previos han diferenciado hasta 8 tipos de metas: metas para la adquisición de competencia y control, metas por el interés en las materias (metas de aprendizaje), metas para una defensa del yo (metas de evitación del rendimiento), metas para una búsqueda de engrandecimiento del yo (metas de aproximación al rendimiento), metas para la adquisición de valoración social, metas de implicación en el estudio por el deseo de obtener un trabajo futuro, metas para evitar castigos (Núñez, González-Pienda, González-Pumariega, García y Roces, 1997; Valle et al., 2006; Valle, Núñez, Cabanach, Rodríguez, González-Pienda y Rosario, 2008), metas por no ser perfectos, metas por no comprender completamente el material o fracasar respecto de sus estándares autorreferidos de dominio (metas de evitación del aprendizaje).

Si bien la orientación a metas puede considerarse un factor esencial a la hora de explicar el proceso de aprendizaje de los estudiantes (Pintrich y Schunk, 2006), a menudo el logro de los objetivos deseados requiere tiempo y esfuerzo, y pueden concurrir situaciones, actitudes y estados que lo dificulten (Husman, McCann y Crowson, 2000). Por ello, en determinadas circunstancias, la habilidad de los estudiantes para poner en marcha estrategias que les ayuden a dirigir su motivación hacia la acción, sin perder de vista el propósito establecido, puede constituirse en un aspecto central del aprendizaje autorregulado.

\section{Activación de creencias de autoeficacia y metas académicas}

Bandura (1997) refiere el proceso por el que los individuos evalúan sus propias experiencias mediante la autorreflexión y desarrollan juicios o creencias personales sobre sus capacidades y logros previos, que ejercerán una fuerte influencia en las actuaciones futuras. En este sentido, los individuos con autoeficacia alta atribuirán sus fracasos a la falta de esfuerzo, lo que potencialmente les llevará a insistir más sobre la tarea y evidenciarán mayor satisfacción personal y autorrealización con los logros, mientras que aquellos que perciben una baja autoeficacia podrían considerar sus fracasos como la evidencia de una falta de habilidad o capacidad 
que incidirá negativamente sobre su compromiso con la tarea. En este sentido, para Bandura (1993) la eficacia percibida interviene como motivador cognitivo.

Probablemente, los alumnos que persiguen y adoptan metas de aprendizaje podrían sostener creencias motivacionales adaptativas respecto a sí mismos y a las tareas de aprendizaje, y podrían emplear la activación de creencias de autoeficacia para sostener su compromiso con la actividad. Del mismo modo, los alumnos orientados a demostrar competencia -metas de aproximación al rendimiento- podrían informar también de procedimientos adaptativos para gestionar su voluntad, utilizando en buena medida estrategias centradas en la activación de creencias de autoeficacia (Montalvo y Torres, 2004).

\section{Demora de la gratificación y metas académicas}

La demora de la gratificación, inicialmente identificada por Bembenutty (1999), se ha considerado una estrategia de gran utilidad para asegurar el éxito en la consecución de las intenciones al facilitar su implementación (Mischel y Shoda, 1995, 1998). Podemos referir la demora de la gratificación como una estrategia basada en el aplazamiento de oportunidades de disponibilidad inmediata para satisfacer los impulsos a favor de perseguir recompensas académicas importantes o las metas elegidas, que son temporalmente remotas, pero mucho más valiosas para el individuo (Bembenutty y Karabenick, 1998). Uno de los estudios llevados a cabo por Bembenutty en 1998 aclaró que existe una relación clara entre la demora de la gratificación y el control de la acción, y, por lo tanto, apoya la idea de que esta estrategia debe ser vista como parte de la red volitiva que los estudiantes activan para asegurar sus intenciones. $\mathrm{La}$ demora de la gratificación asociada con la preferencia diferencial del compromiso con actividades atractivas y actividades poco atractivas pero valiosas, podrían derivarse de una orientación a metas de aprendizaje donde se sostiene un fuerte vínculo entre esfuerzo y resultado (Gaeta, Teruel y Orejudo, 2012).

El objetivo de este estudio es averiguar qué orientación a metas es más característica de los estudiantes que utilizan estrategias volitivas como la demora de la gratificación o la activación de creencias de autoeficacia para el sostenimiento del compromiso con el estudio. Concretamente, se trata de comprobar si existe una asociación positiva entre la orientación al dominio y la vertiente de aproximación de las metas de rendimiento con la utilización de estrategias volitivas como la demora de la gratificación o la activación de creencias de autoeficacia.

\section{Método}

\section{Participantes}

Los participantes en este estudio son estudiantes de 2. curso de educación secundaria obligatoria de un IES público situado en la localidad de Arteixo (La Coruña), ciudad noroccidental en España. El total de participantes en el estudio es de 152, de los cuales el $53,9 \%$ son mujeres y el $46,1 \%$ son varones. La media de edad es de 13,82 años ( $\mathrm{DE}=0,84)$ años, comprendiendo desde los 13 hasta los 16 años.

\section{Instrumentos}

Para conocer cuáles son los principales motivos por los que los estudiantes se esfuerzan en su trabajo académico se empleó el Cuestionario para la evaluación de metas académicas en secundaria (CEMA II) (Núñez et al., 1997). Este cuestionario compuesto por un total de 42 ítems utiliza una escala de tipo Likert de 1 a 5 , ( 1 es nunca y 5 es siempre).

El análisis factorial llevado a cabo sobre las puntuaciones directas de la totalidad de la muestra de este trabajo indica una estructura factorial de 6 factores. Los factores originales de defensa del yo por implicación personal y engrandecimiento del yo no se han diferenciado en el análisis factorial llevado a cabo en este trabajo. Tanto el valor de la medida de adecuación muestral de Kaiser-Meyer-Olkin (KMO) que es de 0,80 , como la prueba de esfericidad de Barlett cuyo nivel de significación es de 0,00 proporcionan respaldo estadístico a la oportunidad de realizar esta factorización. La varianza explicada por los 6 factores extraídos alcanza el 63,09\% del total. La fiabilidad $\alpha$ oscila entre 0,85 y 0,81 correspondientes, respectivamente, a los factores 1 y 2 y 0,70 y 0,77 de los factores 6 y 4 , respectivamente.

Así, este instrumento ha permitido diferenciar los siguientes 4 tipos de metas que, a su vez, contienen 8 conjuntos de razones: a) metas orientadas al aprendizaje (implicación en el estudio para la adquisición de competencia y control; implicación por el interés en las materias); b) metas orientadas al yo (evitación del trabajo derivada de una defensa del yo); c) metas orientadas a la valoración social (implicación derivada de la adquisición de valoración social); d) metas de logro o recompensa (implicación en el estudio por el deseo de obtener un trabajo futuro digno; implicación para evitar castigos).

Para la evaluación de las estrategias motivacionales se ha utilizado el Cuestionario de gestión motivacional (CGM) de Cabanach, Valle, Gerpe, Rodríguez, Piñeiro y Rosario (2009). Se trata de un total de 47 ítems y presenta la misma escala de tipo Likert de 1 a 5.

El CGM diferencia una serie de estrategias volitivas organizadas en 3 escalas: gestión de creencias, de motivos y valor, y de gestión afectiva. Las estrategias relativas a la gestión de las propias creencias serían: la activación de creencias de autoeficacia, la generación de expectativas positivas de resultado y la potenciación del control percibido. Entre las estrategias vinculadas a la gestión de los motivos y el valor, encontramos la evocación de motivos de aprendizaje, evocación de motivos de rendimiento y de motivos de aprobación social, el fomento del valor de la tarea, y la administración de refuerzos extrínsecos. La última de las escalas referida a estrategias de control de la ansiedad, el fomento de la competitividad y la evocación de pensamientos pesimistas no se ha empleado en este trabajo.

El análisis factorial llevado a cabo sobre las puntuaciones directas de la totalidad de la muestra de este trabajo indica, para la Escala de gestión de creencias, una estructura factorial de 2 factores: factor 1_Generación de expectativas positivas de resultado y factor 2 Activación de creencias de autoeficacia (véase tabla 1), en lugar de los 3 correspondientes a la escala original (Cabanach et al., 2009). Tanto el valor de la medida de adecuación muestral de KMO que es de 0,92, como la prueba de esfericidad de Barlett, cuyo nivel de significación es de 0,00 , proporcionan respaldo estadístico a la oportunidad de realizar esta factorización. La varianza explicada por estos 2 factores extraídos a partir de los 13 ítems que finalmente integraron la escala alcanza el $56,67 \%$ del total. La fiabilidad $\alpha$ es de 0,85 para el factor 1_Generación de expectativas positivas de resultado y de 0,83 para el factor 2 Activación de creencias de autoeficacia.

En este trabajo se empleará únicamente como variable la constituida por los 5 ítems integrados bajo es factor 2_Activación de creencias de autoeficacia (véase tabla 1). Ítems como: "Suelo motivarme diciéndome a mí mismo que lo estoy haciendo bien y alabando mi trabajo» (CGM2) o "Me animo diciéndome que conseguiré los resultados deseados» (CGM11) reflejan la habilidad de recordarse a uno mismo que dispone de recursos para afrontar las dificultades.

Del mismo modo se procedió sobre las respuestas de la muestra a la Escala de gestión de los motivos, en el que se han encontrado también algunas discrepancias con respecto a la escala original (Cabanach et al., 2009). Tal y como puede observarse en la tabla 2 este análisis describe una estructura de 4 factores: factor 1_Evocación de motivos de aprendizaje; factor 2_Evocación de motivos 
Tabla 1

Descripción de los ítems y estructura factorial de la Escala de gestión de creencias

\begin{tabular}{|c|c|c|c|c|c|c|c|}
\hline & $\begin{array}{l}\text { Ítem } \\
\text { Encabezado de cada ítem: } \\
\text { Cuando estoy realizando una tarea y me cuesta } \\
\text { mantenerme centrado en el estudio.... }\end{array}$ & M & Asimetría & DT & Curtosis & $\begin{array}{l}\text { Peso factorial } \\
\text { del F1 }\end{array}$ & $\begin{array}{l}\text { Peso factorial } \\
\text { del F2 }\end{array}$ \\
\hline \multirow{8}{*}{$\begin{array}{l}\text { Factor 1: } \\
\text { Generación de } \\
\text { expectativas positivas } \\
\text { de resultado }\end{array}$} & $\begin{array}{l}\text { EGM1: ... me recuerdo que en ocasiones en las que } \\
\text { he tenido que enfrentarme a tareas o asignaturas } \\
\text { dificiles, finalmente no han resultado serlo tanto }\end{array}$ & 3,22 & $-0,24$ & 1,29 & $-0,98$ & 0,79 & $-0,09$ \\
\hline & $\begin{array}{l}\text { EGM4: ... me recuerdo a mí mismo que soy capaz } \\
\text { de esforzarme lo suficiente para tener éxito en la } \\
\text { asignatura a pesar de las dificultades }\end{array}$ & 3,76 & $-0,64$ & 1,17 & $-0,48$ & 0,64 & 0,40 \\
\hline & $\begin{array}{l}\text { EGM6: . . me digo a mí mismo que tengo la } \\
\text { capacidad necesaria para superar la asignatura a } \\
\text { pesar de que me esté encontrando con dificultades }\end{array}$ & 3,61 & $-0,57$ & 1,15 & $-0,51$ & 0,62 & 0,50 \\
\hline & $\begin{array}{l}\text { EGM9: ... me animo a seguir trabajando diciéndome } \\
\text { lo mucho que he avanzado desde que comencé }\end{array}$ & 3,34 & $-0,25$ & 1,26 & $-0,97$ & 0,59 & 0,48 \\
\hline & $\begin{array}{l}\text { EGM7: ... pienso en situaciones similares del pasado } \\
\text { en las que tuve éxito a pesar de las dificultades }\end{array}$ & 3,46 & $-0,49$ & 1,41 & -1.03 & 0,57 & 0,32 \\
\hline & $\begin{array}{l}\text { EGM12: ... me motivo a mí mismo diciéndome que } \\
\text { conseguiré terminar la tarea }\end{array}$ & 3,62 & $-0,61$ & 1,17 & $-0,41$ & 0,55 & 0,54 \\
\hline & $\begin{array}{l}\text { EGM13: ... pienso que las dificultades que me están } \\
\text { desanimando se deben a que no he planteado la tarea } \\
\text { adecuadamente }\end{array}$ & 2,98 & $-0,02$ & 1,13 & $-0,64$ & 0,54 & 0,13 \\
\hline & $\begin{array}{l}\text { EGM3: .. . me convenzo de que los contratiempos en } \\
\text { las tareas no se deben a que no sea un estudiante } \\
\text { capaz, sino a que no lo estoy haciendo correctamente }\end{array}$ & 3,26 & $-0,30$ & 1,20 & $-0,70$ & 0,49 & 0,32 \\
\hline \multirow[t]{5}{*}{$\begin{array}{l}\text { Factor 2: } \\
\text { Activación de creencias } \\
\text { de autoeficacia }\end{array}$} & $\begin{array}{l}\text { EGM8: .. . pienso que en tareas o asignaturas } \\
\text { similares en las que no he sacado una buena nota ha } \\
\text { sido porque no había tenido tiempo de estudiar }\end{array}$ & 2,69 & 0,13 & 1,18 & $-0,76$ & $-0,19$ & 0,78 \\
\hline & EGM10: . . .me motivo a mí mismo dándome ánimos & 3,28 & $-0,28$ & 1,43 & $-1,22$ & 0,48 & 0,67 \\
\hline & $\begin{array}{l}\text { EGM2: ... suelo motivarme diciéndome a mí mismo } \\
\text { que lo estoy haciendo bien y alabando mi trabajo }\end{array}$ & 3,10 & $-0,21$ & 1,41 & $-1,26$ & 0,47 & 0,63 \\
\hline & $\begin{array}{l}\text { EGM5: ... me animo a seguir trabajando diciéndome } \\
\text { que estoy haciendo un buen trabajo }\end{array}$ & 3,38 & $-0,35$ & 1,29 & $-0,94$ & 0,49 & 0,60 \\
\hline & $\begin{array}{l}\text { EGM11: Me animo diciéndome que conseguiré los } \\
\text { resultados deseados }\end{array}$ & 3,45 & $-0,52$ & 1,21 & $-0,60$ & 0,51 & 0,60 \\
\hline Valores propios & & & & & & 6,29 & 1,07 \\
\hline Varianza explicada & & & & & & 30,65 & 26,02 \\
\hline
\end{tabular}

de aprobación social; factor 3_Administración de refuerzos extrínsecos y el factor 4_Fomento del valor de la tarea. Tanto el valor de la medida de adecuación muestral de KMO que es de 0,77, como la prueba de esfericidad de Barlett, cuyo nivel de significación es de 0,00 , proporcionan respaldo estadístico a la oportunidad de realizar dicha factorización. La varianza explicada por los 4 factores extraídos a partir de los 14 ítems que finalmente integraron la escala alcanza el $65,17 \%$ del total. La fiabilidad $\alpha$ oscila entre 0,72 del factor 3_Administración de refuerzos extrínsecos empleado en este trabajo y el 0,75 del factor 1_Evocación de motivos de aprendizaje.

El factor 3 Administración de refuerzos extrínsecos, también referido como demora de la gratificación, integraría ítems como: «Me digo a mí mismo que no haré algo que me gusta si no finalizo el trabajo que tengo que hacer» (CGM15); «Me digo a mí mismo que podré hacer algo que me apetezca si primero hago todo el trabajo planificado» (CGM26) o "Pienso que una vez que termine la tarea o el estudio tendré tiempo libre para otras cosas» (CGM27). Como se puede observar, todas las afirmaciones evidencian la demora de la actividad más apetecible a fin de sostener una meta cuya recompensa se entiende que tendrá mayor peso aún cuando se sitúa a más largo plazo.

\section{Procedimiento}

Una vez obtenida la autorización por parte de la dirección del centro escolar, se administraron los instrumentos de evaluación adaptados a los estudiantes en sus aulas ordinarias en una única sesión y en horario académico. Al comienzo de dicho cuestionario se les insistió a los participantes en que su participación era voluntaria y anónima, y que, por tanto, no tendría ningún tipo de repercusión en sus calificaciones. En ningún momento se vinculó el cuestionario con una determinada asignatura o profesor. Todos los participantes disponían del tiempo que consideraran necesario para leer los ítems detenidamente y responder. El encuestador estuvo presente en todo momento por si surgía alguna duda de comprensión sobre los ítems.

\section{Análisis de datos}

Nuestra investigación se enmarca en una perspectiva empíricoanalítica de carácter cuantitativo con un diseño descriptivo correlacional a través de encuesta. Para el análisis de los datos se recurrió al software estadístico SPSS (IBM SPSS 2.0). Primeramente, se ha realizado un análisis exploratorio de los datos para evaluar su calidad, observar la forma de la distribución, determinar las medidas de resumen y calcular las medidas de dispersión. Para determinar la validez de los instrumentos, se ha realizado un análisis factorial, así como el análisis de contenido y de confiabilidad de los factores. Con objeto de contrastar las hipótesis, y partiendo del análisis de correlación entre las variables de estudio, se empleó el análisis de regresión múltiple por pasos, a fin de determinar el grado en que la estructura de metas predice el uso de estrategias volitivas como la demora de la gratificación o la activación de creencias de autoeficacia.

\section{Resultados}

Atendiendo a los objetivos planteados en este proyecto, se analizarán las relaciones existentes entre las diferentes orientaciones a metas identificadas a partir del CEMAII y 2 de las estrategias 
Tabla 2

Descripción de los ítems y estructura factorial de la Escala de gestión de los motivos

\begin{tabular}{|c|c|c|c|c|c|c|c|c|c|}
\hline & Ítem & M & Asimetría & DT & Curtosis & $\begin{array}{l}\text { Peso factorial } \\
\text { del F1 }\end{array}$ & $\begin{array}{l}\text { Peso factorial } \\
\text { del F2 }\end{array}$ & $\begin{array}{l}\text { Peso factorial } \\
\text { del F3 }\end{array}$ & $\begin{array}{l}\text { Peso factorial } \\
\text { del F4 }\end{array}$ \\
\hline \multirow[t]{5}{*}{$\begin{array}{l}\text { Factor 1: } \\
\text { Evocación de motivos } \\
\text { de aprendizaje }\end{array}$} & $\begin{array}{l}\text { EGM } 30: \text {... me animo a seguir } \\
\text { trabajando pensando en que cuanto } \\
\text { más aprenda, mejor profesional seré }\end{array}$ & 3,6 & $-0,63$ & 1,25 & $-0,62$ & 0,79 & 0,00 & 0,11 & 0,21 \\
\hline & $\begin{array}{l}\text { EGM18: ... pienso que hacer la tarea } \\
\text { y/o estudiar me ayuda a ampliar mis } \\
\text { conocimientos }\end{array}$ & 3,6 & $-0,52$ & 1,19 & $-0,65$ & 0,76 & 0,09 & 0,13 & 0,05 \\
\hline & $\begin{array}{l}\text { EGM23: ... me reto a terminar la tarea } \\
\text { y aprender tanto como pueda }\end{array}$ & 3,2 & $-0,30$ & 1,26 & $-0,83$ & 0,54 & 0,04 & 0,12 & 0,28 \\
\hline & $\begin{array}{l}\text { EGM33: . . me digo a mí mismo que } \\
\text { debería mantenerme trabajando si } \\
\text { realmente quiero dominar lo que estoy } \\
\text { estudiando }\end{array}$ & 3,6 & $-0,42$ & 1,15 & $-0,72$ & 0,69 & 0,09 & 0,11 & 0,13 \\
\hline & $\begin{array}{l}\text { EGM29: ... pienso en el esfuerzo que } \\
\text { está haciendo mi familia para que yo } \\
\text { pueda estar en la universidad }\end{array}$ & 3,7 & $-0,87$ & 1,38 & $-0,51$ & 0,59 & 0,39 & 0,05 & $-0,13$ \\
\hline \multirow[t]{3}{*}{$\begin{array}{l}\text { Factor 2: } \\
\text { Evocación de motivos } \\
\text { de aprobación social }\end{array}$} & $\begin{array}{l}\text { EGM28: ... pienso en lo orgullosos que } \\
\text { se sentirán los demás (familia, } \\
\text { amigos...) si voy bien en los estudios }\end{array}$ & 3,87 & $-0,88$ & 1,26 & $-0,29$ & 0,23 & 0,83 & 0,08 & 0,01 \\
\hline & $\begin{array}{l}\text { EGM16: ... pienso que decepcionaré a } \\
\text { los demás (padres, amigos. ...) si } \\
\text { fracaso en mis estudios }\end{array}$ & 3,48 & $-0,45$ & 1,51 & $-1,29$ & $-0,01$ & 0,82 & 0,09 & 0,12 \\
\hline & $\begin{array}{l}\text { EGM32: ... me obligo a seguir } \\
\text { trabajando pensando en que no quiero } \\
\text { dar una imagen de fracasado ante las } \\
\text { personas importantes para mí }\end{array}$ & 3,35 & $-0,45$ & 1,25 & $-0,80$ & 0,10 & 0,88 & 0,08 & 0,02 \\
\hline \multirow[t]{3}{*}{$\begin{array}{l}\text { Factor 3: } \\
\text { Administración de } \\
\text { refuerzos extrínsecos }\end{array}$} & $\begin{array}{l}\text { EGM26: ... me digo a mí mismo que } \\
\text { podré hacer algo que me apetezca si } \\
\text { primero hago todo el trabajo } \\
\text { planificado }\end{array}$ & 3,81 & $-0,76$ & 1,19 & $-0,30$ & 0,22 & 0,13 & 0,82 & 0,13 \\
\hline & $\begin{array}{l}\text { EGM15: ... me digo a mí mismo que no } \\
\text { haré algo que me gusta si no finalizo } \\
\text { todo el trabajo que tengo que hacer }\end{array}$ & 3,40 & $-0,43$ & 1,30 & $-0,79$ & 0,16 & 0,07 & 0,71 & $-0,08$ \\
\hline & $\begin{array}{l}\text { EGM27: .. . pienso en que una vez que } \\
\text { termine la tarea o el estudio tendré } \\
\text { tiempo libre para otras cosas }\end{array}$ & 4,13 & $-1,36$ & 1,14 & 10,06 & 0,02 & 0,06 & 0,82 & 0,11 \\
\hline \multirow[t]{3}{*}{$\begin{array}{l}\text { Factor 4: } \\
\text { Fomento del valor } \\
\text { de la tarea }\end{array}$} & $\begin{array}{l}\text { EGM21:. . .intento relacionar el } \\
\text { material de estudio o la tarea con algo } \\
\text { que me guste hacer o que encuentre } \\
\text { interesante }\end{array}$ & 3,33 & $-4,6$ & 1,29 & $-0,78$ & 0,09 & 0,12 & $-0,02$ & 0,86 \\
\hline & $\begin{array}{l}\text { EGM20: ... hago que la tarea o el } \\
\text { estudio me resulten más amenos } \\
\text { centrándome en los aspectos más } \\
\text { interesantes o entretenidos }\end{array}$ & 3,43 & $-0,39$ & 1,16 & $-0,58$ & 0,16 & $-0,03$ & 0,14 & 0,78 \\
\hline & $\begin{array}{l}\text { EGM22: ... procuro encontrar alguna } \\
\text { utilidad práctica futura a la tarea o el } \\
\text { material de estudio }\end{array}$ & 3,18 & $-0,09$ & 1,23 & $-0,85$ & 0,53 & 0,06 & 0,02 & 0,59 \\
\hline Valores propios & & & & & & 4,25 & 2,01 & 1,57 & 1,28 \\
\hline Varianza explicada & & & & & & 20,10 & 17,10 & 14,12 & 13,84 \\
\hline
\end{tabular}

En negrita, las cargas factoriales significativas de los ítems por factor.

de autorregulación motivacional evaluadas: la demora de la gratificación o la activación de creencias de autoeficacia (véase tabla 3). Empleando el coeficiente de correlación de Pearson se observó que tanto la demora de la gratificación, referida en consonancia con el

Tabla 3

Coeficientes de correlación de Pearson entre las metas académicas y las estrategias volitivas

\begin{tabular}{lcc}
\hline & $\begin{array}{l}\text { 1E- Activación } \\
\text { de creencias de } \\
\text { autoeficacia 1 }\end{array}$ & $\begin{array}{l}\text { 2E- Demora de } \\
\text { la gratificación }\end{array}$ \\
\hline 3M-Valoración social & $\mathbf{0 , 1 7}$ & 0,06 \\
4M- Evitación de castigos & $-0,02$ & $\mathbf{0 , 2 3}$ \\
5M-Adquisición de trabajo futuro & $\mathbf{0 , 2 7}$ & $\mathbf{0 , 2 7}^{* *}$ \\
6M-Defensa del yo & $-0,14$ & $-0,10$ \\
7M-Interés por la materia & $\mathbf{0 , 3 7}^{* *}$ & $-0,08$ \\
8M-Adquisición de competencia & $\mathbf{0 , 2 4}$ & 0,06 \\
y control & & \\
\hline
\end{tabular}

En negrita, las cargas factoriales significativas.

${ }^{* *}$ La correlación es significativa a nivel 0,01.

* La correlación significativa a nivel 0,05.
CGM original como estrategia de administración de refuerzos externos, como la activación de creencias de autoeficacia sostienen una correlación significativa con la implicación en el estudio para la obtención de un trabajo futuro digno. Sin embargo, parece que únicamente la activación de creencias de autoeficacia correlaciona significativa y positivamente con la implicación por interés en la materia o para la adquisición de competencia y control -metas orientadas al aprendizaje-. Del mismo modo, solo la demora de la gratificación parece mantener una correlación positiva con la implicación para evitar castigos. La búsqueda de aprobación social puede también mantener una correlación positiva de pequeña magnitud con la activación de creencias de autoeficacia.

\section{Orientación a metas y demora de la gratificación}

Profundizando en el análisis de estas relaciones y en consonancia con los objetivos propuestos en este trabajo, se llevó a cabo un análisis de regresión múltiple por pasos considerando la demora de la gratificación a modo de variable dependiente o criterio y las 


\section{Tabla 4}

Coeficientes de correlación múltiple (R), cantidad de varianza explicada por el conjunto de las variables incluidas en el modelo $\left(R^{2}\right)$, y cantidad de varianza explicada por cada una de las variables incluidas en el modelo de predicción (cambio en $\mathrm{R}^{2}$ ) correspondientes a las diferentes orientaciones a metas que predicen las estrategias volitivas de autorregulación del estudio como la demora de la gratificación

\begin{tabular}{lclll}
\hline Modelo & $R$ & $R^{2}$ & $R^{2}$ ajustada & Cambio en $R^{2}$ \\
\hline \multicolumn{2}{l}{$\begin{array}{l}\text { Demora de la gratificación } \\
\text { Modelo 1 }\end{array}$} & & & \\
Modelo 2 & 0,27 & 0,07 & 0,06 & 0,07 \\
\hline
\end{tabular}

Modelo 1: Metas orientadas a la adquisición de un futuro digno.

Modelo 2: Metas orientadas a la adquisición de un futuro digno; metas orientadas a la evitación de castigos.

6 metas diferencias con el CEMA II como las variables predictoras. En la tabla 4 se aporta información sobre las variables del conjunto de predictores que se muestran significativamente relacionadas con la demora de la gratificación, la varianza total explicada por las variables incluidas en el modelo $\left(\mathrm{R}^{2}\right.$ ajustada correspondiente al modelo extraído en el análisis de regresión) así como la cantidad de varianza de demora de la gratificación que explica cada una de las variables incluidas (cambio en $R^{2}$ ).

Para la demora de la gratificación, el modelo correspondiente al último paso indica que las variables predictoras comunes son las metas orientadas a la búsqueda de un futuro digno y a la evitación de castigos, ambas tipificadas como metas vinculadas al logro y las recompensas. La cantidad de varianza explicada del criterio alcanza el $11,7 \%\left(R^{2}\right.$ corregida $\left.=0,11\right)$. Tanto la búsqueda de un futuro digno $(\beta=0,27 ; t=3,55 ; p=0,001)$ como la evitación de castigos $(\beta=0,23 ; t=3,04 ; \mathrm{p}=0,003)$ predecirían positivamente $\mathrm{y}$ en proporciones similares (valor de cambio en $R^{2}$ en la tabla 4 ) el empleo de la demora de la gratificación para gestionar la propia motivación.

\section{Orientación a metas y la activación de creencias de autoeficacia}

De igual modo, profundizando en el análisis de relaciones, se llevó a cabo otro análisis de regresión múltiple por pasos, en este caso, considerando la activación de creencias de autoeficacia como la variable dependiente o criterio, y nuevamente las 6 metas diferenciadas con el CEMA II, como las variables predictoras. En la tabla 5 se muestra la información acerca de las variables del conjunto de predictores que se muestran significativamente relacionadas con la activación de creencias de autoeficacia, la varianza total explicada por las variables incluidas en el modelo $\left(R^{2}\right.$ ajustada correspondiente al modelo extraído en el análisis de regresión) así como la cantidad de varianza de la activación de creencias de autoeficacia que explica cada una de las variables incluidas (cambio en $R^{2}$ ).

\section{Tabla 5}

Coeficientes de correlación múltiple (R), cantidad de varianza explicada por el conjunto de las variables incluidas en el modelo $\left(R^{2}\right)$, y cantidad de varianza explicada por cada una de las variables incluidas en el modelo de predicción (cambio en $\mathrm{R}^{2}$ ) correspondientes a las diferentes orientaciones a metas que predicen la estrategia de activación de creencias de autoeficacia

\begin{tabular}{lccll}
\hline Modelo & $R$ & $R^{2}$ & $R^{2}{ }_{\text {ajustada }}$ & Cambio en $R^{2}$ \\
\hline \multicolumn{4}{l}{ Activación de rreencias de autoeficacia } \\
Modelo 1 & 0,37 & 0,14 & & \\
Modelo 2 & 0,46 & 0,21 & 0,13 & 0,14 \\
Modelo 3 & 0,52 & 0,27 & 0,25 & 0,07 \\
Modelo 4 & 0,55 & 0,30 & 0,28 & 0,05 \\
Modelo 5 & 0,57 & 0,32 & 0,30 & 0,03 \\
\end{tabular}

Modelo 1: M- Interés por la materia.

Modelo 2: M- Interés por la materia; M-Futuro digno.

Modelo 3: M-Interés por la materia; M-Futuro digno; M-Adquisición de control.

Modelo 4: M-Interés por la materia; M-Futuro digno; M-Adquisición de control; M-Valoración social.

Modelo 5: M-Interés por la materia; M-Futuro digno; M-Adquisición de control; M-Valoración social; M-Defensa del yo.
Para la activación de creencias de autoeficacia, el modelo correspondiente al último paso indica, en primer lugar, que las variables predictoras comunes son ordenadas según su potencial predictivo (valor de cambio en $R^{2}$ en la tabla 5), la implicación por interés en la materia, para la búsqueda de un trabajo futuro digno, para la adquisición de competencia y control y de valoración social, así como la evitación del trabajo derivado de la defensa del yo. Tal y como puede observarse en la tabla 5, en este caso, la cantidad de varianza explicada de la activación de creencias de autoeficacia alcanza 30,3\% $\left(R_{\text {corregida }}^{2}=0,30\right)$.

La activación de creencias de autoeficacia vendría positivamente explicada por razones para implicarse como el interés por la materia $(\beta=0,37 ; t=5,52 ; p \leq 0,001)$; la búsqueda de un trabajo futuro digno $(\beta=0,27 ; t=4,00 ; p \leq 0,001)$; la adquisición de competencia y control $(\beta=0,24 ; t=3,54 ; p \leq 0,001)$ y de valoración social $(\beta=0,17$; $t=2,63 ; p=0,009)$. En este caso, se ha encontrado que la evitación del trabajo por defensa de la imagen podría reducir la activación de creencias de autoeficacia como recurso para sostener el compromiso con la actividad $(\beta=-0,14 ; \mathrm{t}=-2,12 ; \mathrm{p}=0,035)$.

\section{Discusión}

Los resultados de esta investigación muestran, en primer lugar, que trabajar por interés en la materia, para lograr un trabajo futuro digno o para ser más capaz y competente puede apoyar la activación de creencias de autoeficacia cuando al alumno le cuesta mantenerse centrado en el estudio. Este resultado permite corroborar básicamente la hipótesis explicativa elaborada para esta estrategia volitiva y enunciada por Montalvo y Torres (2004).

En este punto y con diferente signo, se ha encontrado también una aportación explicativa de la búsqueda de valoración social y desde la orientación a metas egodefensivas. Concretamente, los resultados obtenidos permitirían sostener que esta estrategia volitiva, que esencialmente implica recordarse a uno mismo que se dispone de recursos y habilidades para afrontar las dificultades, podría ser poco empleada cuando se evidencia miedo al fracaso.

Otra de las principales aportaciones de esta investigación gira en torno a la explicación de la demora de la gratificación. A diferencia de lo que se había hipotetizado, y en contradicción con las aportaciones de Abd-El-Fattah y Al-Nabhani (2012) relativas a la contribución positiva de la orientación a metas de dominio, los resultados no muestran una correlación significativa entre esta estrategia motivacional y las metas orientadas a la adquisición de competencia o a las metas centradas en el interés por la materia. $\mathrm{La}$ demora de la gratificación podría estar asociada en mayor medida que otras estrategias volitivas a las metas de logro o recompensa y, específicamente, a la evitación de castigos. Así, los alumnos implicados en el estudio por el deseo de obtener un trabajo futuro digno o para evitar castigos podrían hacer uso de la demora de la gratificación en mayor medida que aquellos caracterizados por una orientación de aprendizaje. Cabe la posibilidad de que los estudiantes que emplean demora de la gratificación se comprometan con su propio aprendizaje porque es importante para sus proyectos o metas futuras, vinculadas tanto a sus estudios (lograr un buen expediente) como a otros aspectos más sociales (conseguir un buen trabajo o vivir bien), sin estar necesariamente interesados -intrínsecamente- en una tarea o en un campo concreto (véase Valle et al., 2006).

Gran parte de la bibliografía referida a la motivación alude a la distinción entre motivación intrínseca y extrínseca (Alonso Tapia, 1995; Huertas, 1997; Lepper, 1988). Parece haber coincidencia entre los distintos autores en vincular la motivación intrínseca con aquellas acciones realizadas por el interés que genera la propia actividad, considerada como un fin en sí misma y no como un medio para alcanzar otras metas. En cambio, la orientación motivacional 
extrínseca se caracteriza generalmente como aquella que lleva al individuo a realizar una determinada acción para satisfacer otros motivos que no están relacionados con la actividad en sí misma, sino más bien con la consecución de otras metas que en el campo escolar suelen fijarse en obtener buenas notas, lograr reconocimiento por parte de los demás, evitar el fracaso o ganar recompensas, entre otras.

Según Alonso Tapia (1995), estas formas de motivación podrían incidir diferencialmente sobre la forma de pensar y, con ello, sobre el aprendizaje desarrollado por el individuo. En este sentido, cabe asumir que distintas orientaciones a metas tendrían consecuencias diferentes sobre el proceso de aprendizaje y, específicamente, sobre la gestión de la motivación en ese proceso. Parece probable que el estudiante motivado intrínsecamente seleccione y realice actividades por el interés, curiosidad y desafío que estas le provocan, y que este alumno motivado intrínsecamente esté más dispuesto a aplicar un esfuerzo mental significativo durante la realización de la tarea, a comprometerse en procesamientos más ricos y elaborados y en el empleo de estrategias de aprendizaje más profundas y efectivas (Lepper, 1988). Mientras, un estudiante motivado por razones más extrínsecas seguramente se comprometerá en las actividades cuando estas ofrecen la posibilidad de obtener recompensas externas o evitar pérdidas, $y$, seguramente, no renunciará a implicarse en tareas fáciles cuya resolución le asegure la obtención de esas recompensas.

Asumiéndolo como parte de la discusión de los resultados, se propone un marco estructural en el que las metas académicas se sitúen en un continuo que se prolonga desde el carácter más interno (autotélico) hasta el carácter más extrínseco con relación al individuo. En este marco, las metas orientadas al aprendizaje serían las metas esencialmente más intrínsecas, ya que trabajar por interés y para adquirir competencia y control serían razones más relativas a la actividad o tarea en sí misma. Estudiar y aprender se observa como un fin en sí mismo. Posiblemente, en una zona más externa de esta estructura, se situarían las metas orientadas al logro o a la recompensa, ya que en este caso el compromiso con la actividad de estudio no está relacionado con esta sino que se consideraría como un medio para alcanzar otros objetivos.

Asumiendo esta organización, y atendiendo a los resultados, cabría considerar la activación de creencias de autoeficacia como una estrategia volitiva capaz de sostener un compromiso más profundo y de mayor calidad con el propio aprendizaje que aquel sostenido mediante la demora de la gratificación. En esta línea, reconociendo las evidencias de que el alumnado con motivación hacia el aprendizaje y el logro presenta unos valores significativamente más altos en rendimiento académico que los alumnos orientados a metas de logro o metas centradas en adquisición de valoración social (Valle et al., 2009), cabe señalar también una asociación importante entre el rendimiento académico y la activación de las creencias de autoeficacia.

En cualquier caso, desde la perspectiva educativa, el compromiso académico está fuertemente vinculado con la motivación y refleja «la voluntad del estudiante de invertir y sostener el esfuerzo en aprender, mientras emplea las necesarias estrategias cognitivas, metacognitivas y volitivas» (Blumenfeld, Kempler y Krajcik, 2006, p. 475). Los aprendices deben tener la voluntad de desarrollar y emplear tales estrategias y, como docentes, debemos considerar como los alumnos se implican en el uso de estrategias de aprendizaje en cada uno de estos 3 niveles. Las estrategias volitivas son las responsables de regular la atención, el afecto y el esfuerzo y, cuando los aprendices las emplean, se fija su toma de decisiones sobre la actividad de aprendizaje y esta se hace más productiva, sensible y efectiva.

Una vez analizados y discutidos los resultados más relevantes de este estudio, y antes de detallar las limitaciones de la investigación, puede ser interesante plantearse algunas preguntas para resolver en futuros trabajos. Quizás, la principal de las cuestiones que se presentan es profundizar en la vinculación diferencial de otras estrategias volitivas y la orientación a metas y estudiar el impacto de estas estrategias volitivas y el rendimiento académico. Por otra parte, consideramos preciso mejorar o construir un instrumento para la evaluación de estrategias volitivas en niveles educativos anteriores al universitario y para otros contextos de logro.

Por último, es necesario mencionar las limitaciones que tiene este proyecto, dadas sus características. Primeramente, es preciso destacar las múltiples razones existentes para ser cautelosos a la hora de generalizar las conclusiones y los resultados presentados. Nuestra muestra es relativamente reducida y la selección del alumnado ha sido por conveniencia, por lo que no podemos cuantificar la representatividad de la muestra. Esta es una de las potenciales causas de que no se hayan extraído las estructuras originales del CEMA II o del CGM (siendo las estructuras obtenidas igualmente válidas y fiables como muestran los índices de KMO). Además, el método de recogida de información ha sido a través de cuestionario, lo que pudo haber desarrollado sesgos en los resultados. Por último, cabe mencionar que el hecho de que sea un estudio transversal provoca que se obtenga la medición de la exposición y del evento de interés en los alumnos en un momento dado. Por este motivo, no es posible determinar una relación de causalidad.

\section{Conflicto de intereses}

Los autores declaran no tener ningún conflicto de intereses.

\section{Referencias bibliográficas}

Abd-El-Fattah, S. M. y AL-Nabhani, H. Z. (2012). From self-theories of intelligence to academic delay of gratification: The mediating role of achievement goals. Australian Journal of Educational \& Developmental Psychology, 12, 93-107 [consultado 13 Oct 2015]. Disponible en: http://files.eric.ed.gov/fulltext/EJ1002250.pdf

Alonso Tapia, J. (1995). Motivación y aprendizaje en el aula. Cómo enseñar a pensar. Madrid: Santillana.

Bandura, A. (1991). Social cognitive theory of self-regulation. Organizational Behavior and Human Decision Processes, 50, 248-287. http://dx.doi.org/10.1016/ 0749-5978(91)90022L

Bandura, A. (1993). Perceived self-efficacy in cognitive development and functioning. Educational Psychologist, 28, 117-148. http://dx.doi.org/10.1207/ s15326985ep2802-3

Bandura, A. (1997). Self-efficacy: The exercise of control. Nueva York: Freeman.

Bembenutty, H. (1999). Sustaining motivation and academic goals: The invaluable role of academic delay of gratification. Paper presented at the annual meeting of the American Educational Research Association, Montreal, Canadá [consultado 12 Oct 2015]. Disponible en http://files.eric.ed.gov/fulltext/ED432511.pdf

Bembenutty, H. y Karabenick, S. (1998). Academic delay of gratification. Learning and Individual Differences, 10, 329-346. http://dx.doi.org/10.1016/ S1041-6080(99)80126-5

Bembenutty, H. y Karabenick, S. A. (2004). Inherent association between academic delay of gratification, future time perspective, and self-regulated learning. Educational Psychology Review, 16, 35-57.

Blumenfeld, P. C., Kempler, T. M. y Krajcik, J. S. (2006). Motivation and cognitive engagement in learning environments. En R. K. Sawyer (Ed.), The Cambridge handbook of the learning sciences (pp. 475-488). New York, NY: Cambridge University Press.

Cabanach, R., Valle, A., Gerpe, M., Rodríguez, S., Piñeiro, I. y Rosario, P. (2009) Diseño y validación de un cuestionario de gestión motivacional. Revista de Psicodidáctica, 14, 29-48. Recuperado de http://recyt.fecyt.es/index.php/REVP/ article/view/6377/5098

Corno, L. (1993). The best-laid plans: Modern conceptions of volition and educational research. Educational Researcher, 22, 14-22.

Corno, L. (2001). Volitional aspects of self-regulated learning. En B. J. Zimmerman y D. H. Schunk (Eds.), Self-regulated learning and academic achievement: Theoretical perspectives 2 (pp. 191-225). Hillsdale, NJ: Erlbaum.

Gaeta, M., Teruel, M. y Orejudo, S. (2012). Aspectos motivacionales, volitivos y metacognitivos del aprendizaje autorregulado. Electronic Journal of Research, 10, 73-94. Nueva York: Routledge [consultado 12 Oct 2015]. Disponible en http://www.investigacion-psicopedagogica.org/revista/new/ContadorArticulo. php?640

García, T. (1995). The role of motivational strategies in self-regultated learning. En P. Pintrich (Ed.), Undertanding self-regulated learning.. San Francisco: Jossey-Bass Publishers. 
Graham, S. y Harris, K. (1997). Self-regulation and writing: Where do we go from here? Contemporary Educational Psychology, 22, 102-114. http://dx.doi.org/ 10.1006/ceps.1997.0920

Huertas, J. (1997). Motivación. Querer aprender. Buenos Aires: Aique.

Husman, J., McCann, E. y Crowson, H. (2000). Volitional strategies and future time perspective: Embracing the complexity of dynamic interactions. International Journal of Educational Research, 33, 777-799. http://dx.doi.org/10.1016/ S0883-0355(00)00050-1

Kaplan, A. (2008). Clarifying metacognition, self-regulation, and self-regulated learning: What's the purpose? Educational Psychology Review, 20, 477-484. http://dx.doi.org/10.1007/s10648-008-9087-2

Lepper, M. (1988). Motivational considerations in the study of instruction. Cognition and Instruction, 5, 289-309. http://dx.doi.org/10.1207/s1532690xci0504_3

López, D. (2000). Social cognitive influences on self-regulated learning: The impact of action-control beliefs and academic goals on achievement-related outcomes. Learning and Individual Differences, 11, 301-319. http://dx.doi.org/10.1016/ S1041-6080(99)80005-3

Mischel, W. y Shoda, Y. (1995). A cognitive-affective system theory of personality: Reconceptualizing situations, dispositions, dynamics, and invariance in personality structure. Psychological Review, 102, 246-268. http://dx.doi.org/10.1037/ 0033-295X.102.2.246

Mischel, W. y Shoda, Y.(1998). Reconciling processing dynamics and personality dispositions. Annual Review of Psychology, 49, 229-258 [consultado 12 Oct 2015]. Disponible en http://data.psych.udel.edu/abelcher/Shared\%20Documents/ 2\%20Personality,\%20Emotion,\%20and\%20Individual\%20Differences\%20(17)/ Mischel,\%20Shoda,\%201998.pdf

Montalvo, F. y Torres, M. (2004). El aprendizaje autorregulado: presente y futuro de la investigación. Electronic Journal of Research in Educational Psychology, 2, 1-34 [consultado 12 Oct 2015]. Disponible en http://www.investigacionpsicopedagogica.org/revista/articulos/3/espannol/Art_3_27.pdf

Núñez, J. C., González-Pienda, J. A., González-Pumariega, S., García, M. y Roces, C. (1997). Cuestionario para la evaluación de metas académicas en secundaria (CEMAII). Oviedo: Departamento de Psicología, Universidad de Oviedo.

Pintrich, P. (2000). Taking control of research on volitional control: challenges for future theory and research. Learning and Individual Differences. 11, 335-354. http://dx.doi.org/10.1016/S1041-6080(99)80007-7
Pintrich, P. y de Groot, E. (1990). Motivational and self-regulated learning component of classroom academic performance. Journal of Educational Psychology, 82, 33-40. http://dx.doi.org/10.1037/0022-0663.82.1.33

Pintrich, P.y Schunk, D. (2006). Motivación en contextos educativos. (M. Limon Trad.) Madrid: Prentice Hall.

Rodríguez, S., Cabanach, R., Valle, A., Núñez, J. y González-Pienda, J. (2004). Diferencias en el uso del self-handicapping y pesimismo defensivo y sus relaciones con las metas de logro, la autoestima y las estrategias de autorregulación del aprendizaje. Psicothema, 16, 626-632 [consultado 12 Oct 2015]. Disponible en http://www.psicothema.com/pdf/3042.pdf

Urdan, T. (2004). Predictors of academic self-handicapping and achievement: Examining achievement goals, classroom goal structures, and culture. Journal of Educational Psychology, 96, 251-264. http://dx.doi.org/10.1037/0022-0663.96.2.251

Valle, A., Cabanach, R., Rodríguez, S., Núñez, J. y González-Pienda, J. (2006) Metas académicas, estrategias cognitivas y estrategias de autorregulación del estudio. Psicothema, 18, 165-170 [consultado 12 Oct 2015]. Disponible en http://www.unioviedo.net/reunido/index.php/PST/article/view/8488

Valle, A., Núñez, J., Cabanach, R., Rodríguez, S., González-Pienda, J. y Rosario, P. (2008). Capacidad predictiva de las metas académicas sobre el rendimiento en diferentes áreas curriculares. Revista Latinoamericana de Psicología, 40 111-122 [consultado 12 Oct 2015]. Disponible en http://www.scielo.org.co/ scielo.php?pid=S0120-05342008000100009\&script=sci_abstract

Valle, A., Núñez, J., Cabanach, R., Rodríguez, S., González-Pienda, J. y Rosario, P. (2009). Perfiles motivacionales en estudiantes de secundaria: análisis diferencial en estrategias cognitivas, estrategias de autorregulación y rendimiento académico. Revista Mexicana de Psicología, 26, 113-124 [consultado 12 Oct 2015]. Disponible en http://hdl.handle.net/1822/11860

Wolters, C. A. (1999). The relation between high school students' motivational regulation and their use of learning strategies, effort, and classroom performance. Learning and individual differences, 11, 281-299. http://dx.doi.org/10.1016/ S0883-0355(00)00051-3

Wolters, C. A. (2003). Regulation of motivation: Evaluating an underemphasized aspect of self-regulated learning. Educational psychologist, 38, 189-205. http://dx.doi.org/10.1207/S15326985EP3804_1

Zimmerman, B.y Schunk, D. (1998). Self-regulated learning. Nueva York: The Guilford Press. 